\title{
"Image Compression analysis and evaluation for various image sequences in reference to intra prediction"
}

\author{
Akhilesh Latoria $^{1}$, DB Ojha ${ }^{2}$ \\ Research Scholar, Dept. Computer Science., Mewar University, Chittorgarh, India ${ }^{1}$ \\ Dept. Mathematics., Mewar University, Chittorgarh, India ${ }^{2}$
}

\begin{abstract}
The present still picture compression method does not have the required level of institutionalization and still have something can be enhance as per compression rate, calculation, etc. This paper utilizes the strategy of H.264/MPEG-4 Advanced Video Coding to enhance still picture compression. The H.264/MPEG-4 standard guarantees much higher compression and quality contrasted with other existing standard, for example, MPEG-4 and H.263. This paper uses the intra expectation methodology of H.264/AVC and Huffman coding to enhance the compression rate. Each $4 \times 4$ piece is anticipated by picking the best mode out of the 9 diverse modes. The best forecast mode is chosen by Sum of Absolute Error technique. Likewise this paper manages a picture concealing calculation based on particular quality disintegration calculation. This paper propose an information concealing algorithm , applying on encoded bitstream.
\end{abstract}

Keywords: Intra Prediction, Picture Compression, Huffman Coding, Singular Value Decomposition Peak Signal to Commotion Ratio,H.264,JPEG.

\section{INTRODUCTION}

As of late, compressed pictures have turned into the most enhance picture compression. Intra forecast is a successful well known on the web, essentially on the grounds that technique for decreasing the repetition of a picture. So the they take less space than other crude pictures [3]. additional information in a picture is lessened. At that H.264/AVC is the video coding standard of the ITU-T point perform entropy disentangling as in H264 standard, Video Coding Experts Group furthermore, the ISO/IEC yet as opposed to utilizing the variable length coding Moving Picture Experts Group. The primary objectives of Huffman coding [8] is utilized .Huffman coding is a the H.264/AVC have been upgraded compression lossless information concealing algorithm and can repeat execution and procurement of a system well disposed at the recipient side without much degradation.

video representation.H.264/AVC has accomplished critical change in rate-bending effectiveness with respect to existing models [20].

Picture compression is a very much concentrated on theme that codes the pictures into less measures of information. There are two sorts of picture compression approaches: lossless and lossy. Lossless picture pressure methods are without mistake coding strategies. A lossless-packed picture can be decompressed to be one which is indistinguishable to the first picture. Since lossless pressure strategies keep point by point data in the picture, the sizes of the compacted results are not decreased to such an extent. Lossy picture pressure methods rather deliver results with littler sizes and the picture acquired from decompressing is not indistinguishable to the unique one [8].

A large portion of the past work on compressed space picture watermarking concentrated on implanting the watermark into the JPEG bit stream. Here intra forecast ofH.264 pressure standard [9] is received. The residual obstructs in the H.264 standard are compressed utilizing the Discrete cosine transform change, quantized, reordered, run level coded, and afterward entropy encoding is connected. This paper embraces the system of intra expectation methodology of H264/AVC is utilized to

The point of a lossy compression system is to getting higher similitude to the first picture and keeping up higher compression rates. Lossy compression as the name suggests results in loss of some data, that compresses information by tossing (losing) some of it. Lossy picture compression is valuable in applications, for example, show $\mathrm{TV}$, videoconferencing, and copy transmission, in which a sure measure of blunder is an adequate exchange off for expanded compression execution.

These days, just couple of arrangements are proposed for both access to the spread picture and mystery picture with adequate quality. The Current techniques for the implanting of information into the spread picture fall into two classifications: spatial based plans furthermore, change based plans. Spatial based plans implant the information into the pixels of the spread picture specifically with no visual changes, while change based plans implant the information into the spread picture by changing the coefficients in a change area, for example, the DCT and wavelet changes. In the proposed system the deposit of spread picture is encoded first and afterward the mystery picture is implanted. Spatial space calculations have the advantage in steganography limit, however the burden is frail robustness. Change area algorithm is implanting the mystery data in the transform space. This 
sort of algorithms has the upside of good soundness, be that as it may, the burden of little limit [17].The mystery information is implanted into the quantized coefficients before entropy encoding [10]. This paper centers upon information covering up after entropy encoding. For picture implanting Singular Esteem Decomposition calculation is utilized.

This paper is sorted out as takes after: In Section II the Intra expectation based picture compression procedure is presented. Segment III displays the proposed methodology of information concealing strategy. Area IV depicts the framework model. Segment V and gives test comes about and area VI portrays examinations and future work. At last, the paper is finished up in Section VII.

\section{INTRA PREDICTION BASED PICTURE COMPRESSION AND DECOMPRESSION}

\section{PROCEDURE}

The intra prediction based compression technique proposed for H.264/AVC video coding standard by ITU-T Video Coding Experts Group and the ISO/IEC Moving Picture Specialists Group [12]. In the proposed approach, the intra prediction methodology of H.264/AVC and Huffman coding received for picture coding to enhance the compression rate.

MPEG-4 is a state-of-art video coder with many advanced features, which were not available in its predecessors such as MPEG-1/2. MPEG-4 combines some typical features of other MPEG standards, but aims to provide a set of technologies to satisfy the needs authors, service providers and end users. It enables much functionality potential accessible on a single terminal and higher levels of interaction with content. MPEG-4 achieves these goals by providing standardized ways to support: coding, composition, multiplexing and interaction[3,4]. The key feature of MPEG-4 is decompose a video frames in different layers of video object plane (VOP). Figure 1 Demonstrate the Picture compression technique.

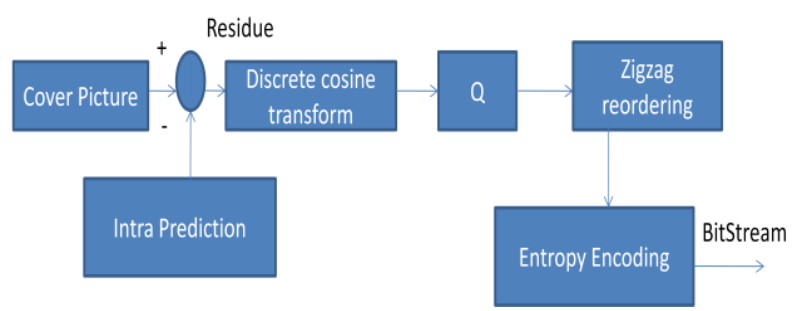

Fig 1: Schematic diagram of Image Compression

The goal of intra prediction in picture pressure is to lessen spatial redundancies between contiguous pixels so better compression proportion can be accomplished. The intra indicator is utilized before Discrete cosine transform block. Intra forecast is a powerful technique for lessening the repetition of a picture or an intraframe. For encoding a block or full scale hinder in Intra-coded mode, an expectation piece is shaped taking into account already recreated pieces. In H.264/AVC, the prediction square may be framed for every $4 \times 4$ sub obstruct, every $8 \times 8$ square, or for a $16 \times 16$ large scale block[6]. For a 4 x 4 sub square, nine intra expectation modes are characterized. i.e. there are nine distinctive directional methods for performing the expectation, The nine intra prediction modes named $0,1,3,4,5,6,7$, furthermore, 8 . The bolts in Figure 2 demonstrate the course of prediction in every mode. Mode 2 is "Discrete cosine-prediction". The different modes speak to headings of predictions as showed in Figure2

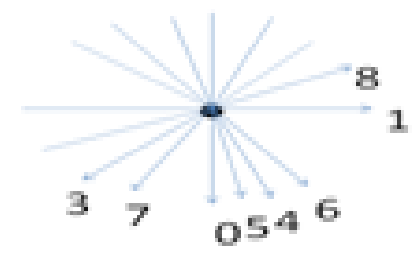

Fig 2: Directions of $4 * 4$ and $8 * 8$ intra prediction modes

$$
0,1 \text {, and } 3-8 \text {. }
$$

The encoder chooses the prediction mode for every piece that minimizes the remaining in the middle of $\mathrm{P}$ and the block to be encoded. The 9 prediction modes $(0-8)$ are figured for the $4 \times 4$ block. The Sum of Absolute Errors (SAE) for each prediction demonstrates the size of the prediction error.The best match to the real current block is distinguished as per the littlest SAE (Sum of Absolute Error) [20,12, and 6].

The residual of the expectation, which is the distinction between the first and the anticipated block, is changed. The change coefficients are scaled and quantized. The quantized change coefficients are entropy coded and transmitted. Here buildup picture is encoded utilizing Huffman coding. In this manner these qualities are changed over into paired codes utilizing Huffman coding. This encoding system delivers an productive, reduced twofold representation of the data. The encoded bit stream can then be put away. Huffman coding improves the security by method for encoding. Huffman encoded bit stream can't uncover anything. To remove meaning, the Huffman table is required to translate. It gives one kind of confirmation, as any single piece change in the Huffman coded bit stream, Huffman table is not able to decipher.

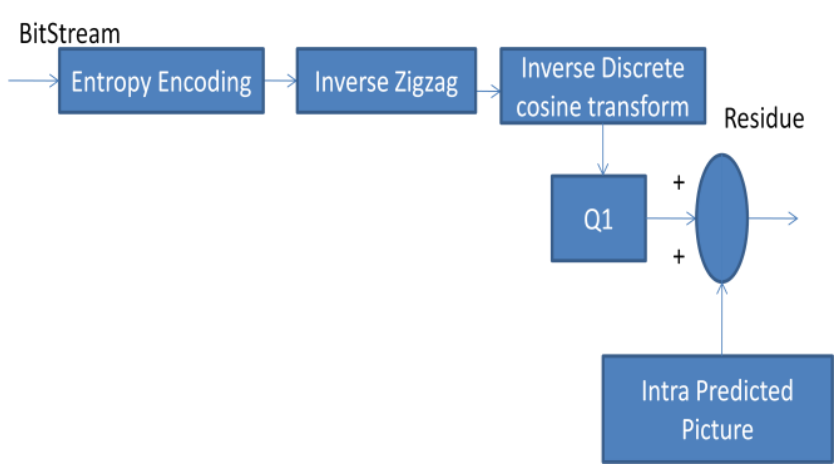

Fig 3: Schematic diagram of image decompression

Figure 3 demonstrates schematic diagram of picture decompression. The decoder translates each of the linguistic structure components and extracts the data portrayed above, i.e. quantized change coefficients, expectation data and so on. This data is at that point used to turn around the coding handle and reproduce the pictures. The last remade picture is recreated by including intra predicted picture. 


\section{ADVANCED APPLICATION OF PICTURE HIDING AND EXTRACTION METHOD}

This segment presents the concealing and extraction technique. The concealing information is inserted in the bit stream of the host picture. The host picture is a dim scale picture and the secret picture is the shading picture. All stenography methods are connected specifically to the cover pictures. The cover picture is encoded and frames the bit-stream. The picture covering up is taking into account the Singular Value Decomposition Calculation [2].

\section{The Embedding Technique}

The idea of the proposed picture hiding technique is shown in figure 4. An exceptionally basic embedding algorithm is utilized, that adjusts the encoded bit stream. The algorithm installs the secret picture by altering inclining components of the host picture. This technique creates an embedded picture.

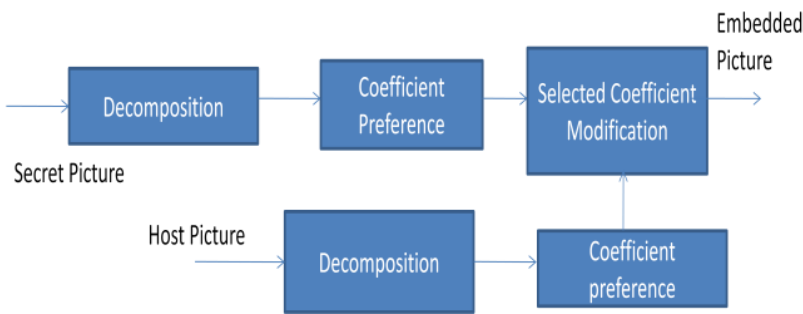

Fig IV: Embedded Secret Picture in a Compressed picture

The progressions of embedding the secret picture can be condensed as takes after:

Step I : Select secret picture.

Step II: Use level one Discrete Wavelet Transform on secret picture.

Step III: Perform Singular Value Decomposition Algorithm and select the bits to be embedded.

StepIV: Choose the encoded bit stream and resize the bit stream to generate a host image.

StepV:-Apply level one Discrete Wavelet Transform on host image.

StepVI:- Apply Singular Value Decomposition in to three sub bands $\mathrm{LH}, \mathrm{HL}$ and $\mathrm{HH}$ of the host picture.

Singular Value Decomposition Algorithm, then host image decomposes into three matrices.

$$
\mathrm{M}=\mathrm{U}[\mathrm{mxm}] * \mathrm{~S}[\mathrm{mxn}] * \mathrm{VK}[\mathrm{nxn}]
$$

Where the $\mathrm{M}$ is a host image, $\mathrm{U}$ is a unitary matrix, $\mathrm{S}$ matrix is a diagonal matrix with non-negative real numbers on the diagonal and the $\mathrm{V}$ matrix is a $\mathrm{n} * \mathrm{n}$ unitary matrix over K consists of set of 'right' orthonormal bases. Select the $\mathrm{S}$ matrix and embed one bit in the $\mathrm{S}$ matrix according to the equation (A).

$$
\mathrm{Si} 1=\mathrm{Si}+\mathrm{k} * \mathrm{~b}
$$

Where Si : Actual Coefficients

$$
\begin{aligned}
& \mathrm{S}_{\mathrm{i}}{ }^{1} \text { : Marked Coefficients } \\
& \mathrm{b} \text { : Bit to be embedded } \\
& \mathrm{k} \text { : Watermark Strength }
\end{aligned}
$$

Subsequent embedding the watermarked picture is gotten by the mathematical statement (B).

$$
\text { Embedded Image }=\mathrm{U} * \mathrm{~S} 1 * \mathrm{VT}(\mathrm{B})
$$

StepVII: Apply Inverse Discrete Wavelet Transform and shapes the embedded picture.

\section{Extraction Technique}

The information extraction methodology are like that of the embedding methodoly. Figure 5 demonstrates the piece outline of separating secret picture from the embedded picture.

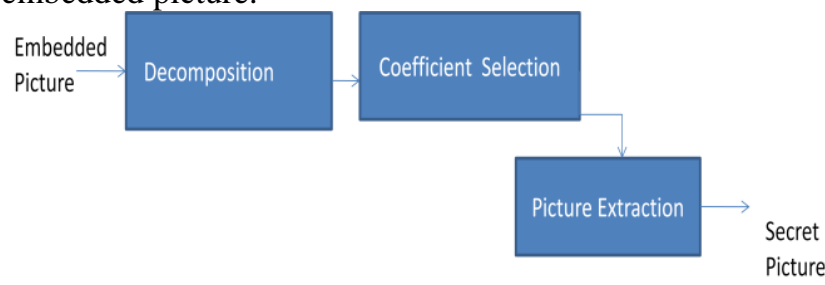

Fig. V: Extracting Secret Picture from the Embedded Picture

The extracting procedure can be all the more definitely portrayed by the strides:

Step I: Choose the embedded picture

Step II: Perform Singular Value Decomposition to get the Marked Coefficients.

Step III: By doing the reverse operation, unique values are gotten, At that point get the secret picture by the comparison (B)

$$
\mathrm{b}=\mathrm{Si} 1-\mathrm{Si} / \mathrm{k}
$$

\section{FRAMEWORK MODEL}

This area depicts how this codec performs. The extraction of the concealed picture and reproduction of the cover picture should be possible differently. The first comprises to remake the message from the coded bit stream amid the decompression stage. The Second one comprises to recover the concealed message from the embedded picture. In this paper, the transmission stage is performed in the wake of checking private key by the framework encoder. Picture Compression and Embedding System Encoder

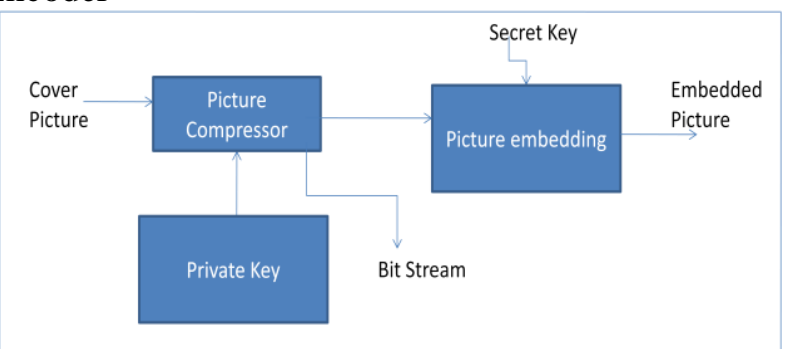

Fig 6: Picture Encoding and Embedding Schematic Diagram

Keeping in mind the end goal to get the compressed picture, intra prediction based picture compression is utilized .For picture embedding Singular Value Decomposition Algorithm is utilized. Therefore there are two Essential parts, cover picture and concealing information. Therefore there are two various types of blocks marked and unmarked blocks. Figure 6 demonstrates the picture encoding and inserting block diagram.

Before encoding, the secret key is created by the encoder to affirm the security. This secret key is one time private 
key. The one time private key is to be utilized just once, and quickly disposed of. Every client will dependably produce a new private key. Once the private key is utilized or when it is lapsed with the session, the private key is deleted and discarded.

Encoder first looks for the security code, before transmitting any information to the decoder i.e., the proposed encoding process looks at the decoder key to decide the transmission code. On the off chance that the key is superbly coordinating to the decoder key, the transmission code is the embedded picture. So just the indented client can apply the extracting algorithm to recover secret picture. In the other case, if the decoder key is most certainly not coordinating to the encoder key, the transmission code is the compressed picture. The Deciphering algorithm is performed to recreate the cover picture.

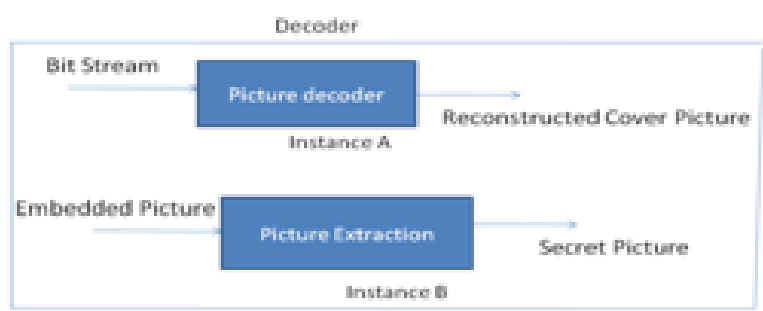

Fig 7: Picture Decoding and Extracting Schematic Diagram

\section{TEST PERFORMED}

In this segment, a few examinations are done to demonstrate the efficiency of the proposed plan. The proposed system has been reproduced utilizing the MATLAB 7.7 project on Windows 7 . An arrangement of 8-bit pictures are utilized as the cover picture. The shading pictures of distinctive measurements are hiding away on these pictures.

For this analysis, 4 cover pictures are utilized, these are appeared in Figure 5. A lossless compression was connected to the cover pictures.

The shading pictures of diverse measurements are covering up on these dark pictures. The parameters considered for assessment are as per the following:

1. Compression Ratio: The compression is to diminish the quantity of bits the picture is spoken to by. Picture compression is measured by the means of an index called Compression Ratio:

$$
\mathrm{CR}=\mathrm{n} 1 / \mathrm{n} 2
$$

Where, $\mathrm{n} 1$ is the quantity of bits in the first picture. While $\mathrm{n} 2$ is the quantity of bits acquired after the compression. There are two sorts of compression: lossy and lossless compression [19].In lossy compression the data is saving however has low compression proportions. Lossy compression gives abnormal state of information decrease, where loss can be endured.

2. PSNR: PSNR remains for Peak Signal to Noise Ratio and it is ascertained utilizing the accompanying mathematical statement:

$$
\text { PSNR=10 log } 10 \text { 2552/MSE }
$$
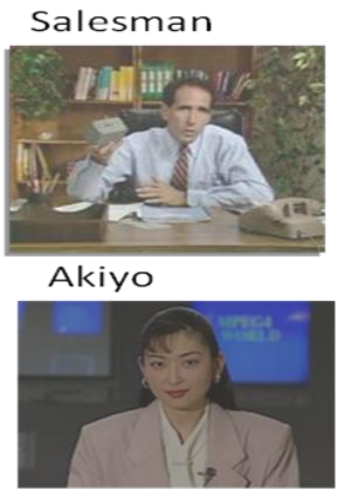

Fig 8: Cover Pictures

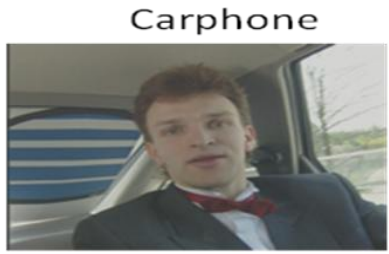

Foreman

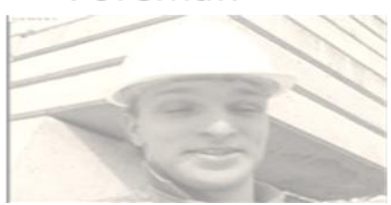

The Mean Square Error (MSE) is the estimation of average of square of errors and the combined squared error of noisy and original picture.

A higher estimation of PSNR is great due to the prevalence of the signal over that of the noise [10]

\section{Compression Ratios:}

By test comes about, the proposed system has better impacts taking into account the compression ratio while keeping up great Peak Signal to Noise Ratio when reproduced. Figure 8 demonstrates the reconstructed cover pictures.
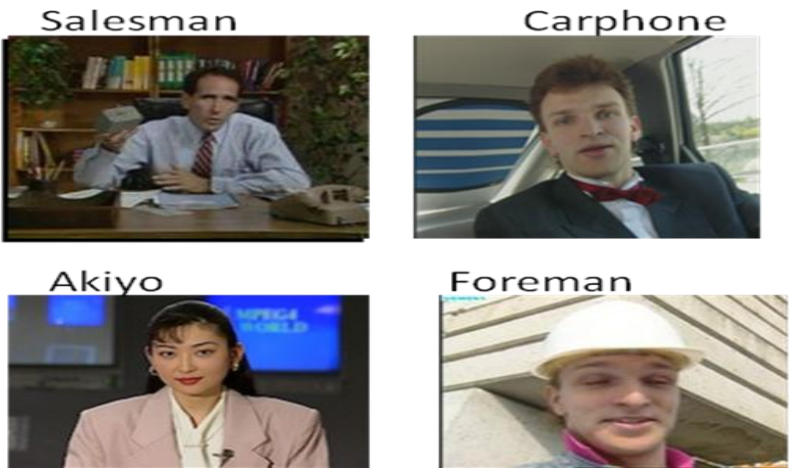

Fig 9: Reconstructed Cover Pictures

Table 1 demonstrate the Compression Ratio, Relative Data Duplicity, Peak Signal to Noise Ratio of different test pictures.

Table 1: Result of Intra prediction picture compression

\begin{tabular}{|l|l|l|}
\hline Test Pictures & Compression Ratio & PSNR \\
\hline Salesman & 11.67 & 31.6 \\
\hline Carphone & 39.95 & 30.29 \\
\hline Akiyo & 58.67 & 31.01 \\
\hline Foreman & 37.87 & 32.01 \\
\hline
\end{tabular}

Table 2 : Comparison of Peak Signal to Noise ratio of original image and Reconstructed Image.

\begin{tabular}{|l|l|l|l|}
\hline $\begin{array}{l}\text { Test } \\
\text { Pictures }\end{array}$ & JPEG & H.264 & $\begin{array}{l}\text { Proposed } \\
\text { Method }\end{array}$ \\
\hline Salesman & 28.202 & 32.15 & 32.31 \\
\hline Carphone & 32.827 & 33.11 & 29.43 \\
\hline Akiyo & 33.218 & 34.30 & 31.98 \\
\hline Foreman & 32.453 & 34.34 & 34.25 \\
\hline
\end{tabular}


Table 3 : Comparison of Compression Ratios

\begin{tabular}{|l|l|l|l|}
\hline $\begin{array}{l}\text { Test } \\
\text { Pictures }\end{array}$ & JPEG & H.264 & $\begin{array}{l}\text { Proposed } \\
\text { Method }\end{array}$ \\
\hline Salesman & 9.035 & 8.981 & 12.668 \\
\hline Carphone & 8.568 & 8.561 & 40.249 \\
\hline Akiyo & 8.138 & 9.286 & 59.641 \\
\hline Foreman & 9.002 & 9.003 & 38.461 \\
\hline
\end{tabular}

While considering the estimation values from the table 2 and table 3 , it is clearly clear that the pressure proportion delivered by the proposed strategy was bigger than that of the JPEG and H.264 compression measures. In this way the proposed system can accomplish great compression ratio without recognizable degradation in PSNR. Test results demonstrate that the proposed calculation can enhance the compression rate up to $35 \%$ averagely contrasting with the JPEG and H.264 compression norms.

\section{FUTURE WORK AND DISCUSSIONS}

This paper introduces an extremely straightforward embedded algorithm that alters the encoded bit stream. The proposed system has insignificant impact on the nature of the cover picture while recreated. The utilization one time private key is the quality of the strategy. The quality of the strategy is that uses a one time private key .This secret picture figures out which code is to be transmitted. The future work go for making more advanced stenographic strategy for concealing secret picture without unmistakable change in the cover picture in order to make embedding identification is unthinkable. In this way proposed method can accomplish large compression ratio without noticeable degradation in PSNR. Experiment result shows that the proposed algorithm can amplify the compression rate up to certain extent as compare to JPEG and MPEG4.

\section{CONCLUSION}

For the most part, picture stegnography strategy does not give much consideration on the compression proportion. In this paper, the cover picture is compacted and frame a bit stream and after that the secret picture is stowing away on this bit stream. By enhancing a current encoding strategy, the security of the embedded picture can be enhanced while keeping the same robustness of the first technique. The one time private key figures out which code is to be transmitted. So this operation gives adequate secrecy. As per the test comes out the proposed strategy has higher compression proportion at an expense of slight chance in Peak signal to noise ratio.

\section{REFERENCES}

[1] Ali Al-Ataby and Fawzi Al-Naima, "A Modified High Capacity Image Steganography Technique Based on Wavelet Transform", The International Arab Journal of Information Technology, Vol.7, No. 4, October 2010

[2] Andrews, H. Patterson, C., "Singular Value Decomposition (SVD) Image Coding", Acoustics, Speech and Signal Processing, IEEE Transactions on Image Processing. Volume: 24, Issue: 1,1976.

[3] Ayman Abdalla, Ahmad Mazhar, Mosa Salah, Sahar Khalaf "Comparative study of compression techniques for synthetic videos" ,The International Journal of Multimedia \& Its Applications(IJMA) Vol.6, No.2, April 2014

[4] R. A. Ghazy, M. M. Hadhoud, M. I. Dessouky, N. A. El-Fishawy, F. E. Abd El-Samie, "Performance Evaluation Of Block Based Svd Image Watermarking", Department of Electronics and Electrical Communications ,Faculty of Electronic Engineering,Menoufia University,32952, Menouf, Egypt.

[5] Charilaos Christopoulos, Touradj Ebrahimi," The JPEG2000 Still Image Coding System: An Overview", IEEE Transactions on Consumer Electronics. Vol. 46, No. 4, pp. 1103-1127, November 2000.

[6] Chen Chen and Ping-Hao Wu, Homer Chen, "Transform-Domain Intra Prediction for H.264", National Science Council,0-78038834-8/05/\$20.00 @2005 IEEE.

[7] Da-Chun Wu' and Wen-Hsiang Tsai," Data Hiding In Images Via Multiple-Based Er Conversion And Lossy Compression", IEEE Transactions on Consumer Electronics, Vol. 44, No. 4, November 1998.

[8] D. A. Huffman, "A method for the construction of minimum redundancy codes,'Proceedings IRE, vol.40, pp. 1098-1101, 1952.

[9] Hsien-Wen Tseng, Chin-Chen Chang," High Capacity Data Hiding in JPEG-CompressedImages", Informatica, Institute of Mathematics and Informatics, Vilnius 2004, Vol. 15, No. 1, 1271422004.

[10] Hsien-Wen Tseng, Chin-Chen Chang," High Capacity Data Hiding in JPEG-Compressed Images", NFORMATICA, 2004, Vol. 15, No. 1, Institute of Mathematics and Informatics, Vilnius, 2003.

[11] "H.264 video compression standard", White paper, Axis communications, 2002.

[12] Iain Richardson, Vcodex, "H.264/ AVC Intra Prediction". www.vcodex.com,2010 .

[13] K B Shiva Kumar, K B Raja,R K Chhotaray, Sabyasachi Pattanaik," Bit Length Replacement Steganography based on DCT Coefficients", International Journal of Engineering Science and Technology Vol. 2(8), 2010, 3561-3570

[14] N. F. Johnson and S. Katzenbeisser," .A survey of steganographic techniques", in S. Katzenbeisser and F. Peticolas (Eds.): Information Hiding, pp.43-78. Artech House, Norwood, MA, 2000.

[15] Mahmud Hasan, Kamruddin Md. Nur, Tanzeem Bin Noor," A Novel Compressed Domain Technique of Reversible Steganography"., International Journal of Advanced Research in Computer Science and Software Engineering., Volume 2, Issue 3, March 2012.

[16] Majid Rabbani, Rajan Joshi "An overview of the JPEG2000 still image compression standard", Signal Processing: Image Communication 17 (2002) 3-48.

*[17] Mohanbaabu G and P. Renuga," Still Image Compression Using Texture and Non Texture Prediction Model", American Journal of Applied Sciences 9 (4): 519-525, 2012 ISSN 1546-9239 (C) 2012 Science Publications

[18] Pankaj Topiwala"Comparative Study of JPEG 2000 and H.264/AVC FRExt I- Frame Coding on High- Definition Video Sequences", FastVDO LLC, 7150 Riverwood Dr., Columbia, Maryland, 21046-1245 USA,2005.

[9] Po-Yueh Chen and Hung-Ju Lin, "A DWT Based Approach for Image Steganography", International Journal of Applied Science and Engineering 2006. 4, 3: 275-290.

[19] Sang-Gyu Cho, Zoran Bojkovi'c, Dragorad Milovanovi'c, Jungsik Lee, and Jae-Jeong Hwang "Image Quality Evaluation: JPEG 2000 Versus Intra-only H.264/AVC High Profile", FACTA UNIVERSITATIS (NIS) SER.: ELEC. ENERG. vol. 20, no. 1, April 2007, 71-83

[20] "Video coding for low bit rate communication," Int. Telecommun.Union-Telecommun. (ITU-T), Recommendation H.263, version 1,1995; version 2,1998; version 3, 2000. 